memo (2009) Vol. 2: 63

DOI 10.1007/s12254-009-0122-x

Printed in Austria

(C) Springer-Verlag 2009

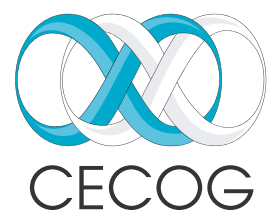

Central European Cooperative Oncology Group

\title{
Initiation of the TURANDOT breast cancer study
}

The following CECOG-protocol is open for patient enrolment:

\section{CECOG/BC.1.3.005 - TURANDOT}

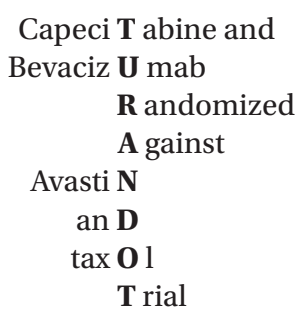

A randomized phase III 2-arm trial of paclitaxel plus bevacizumab vs. capecitabine plus bevacizumab for the first-line treatment of HER2-negative locally recurrent or metastatic breast cancer.

Principal Investigator: Vice-Coordinator:

Sponsor:
Prof. Christoph Zielinski, M.D.

Thomas Brodowicz, M.D., Associate Professor CECOG
Participating countries: Austria, Bosnia, Bulgaria, Croatia, Czech Republic, Hungary, Israel, Latvia, Poland, Romania, Serbia and Slovakia.

This study requires $\mathbf{4 9 0}$ eligible patients. Assuming an exclusion rate of up to $12 \%$ from the per-protocol population, 560 eligible patients need to be recruited. The recruitment of patients started in September 2008.

If you are interested to learn more about this trial, please contact CECOG, attn. Dagmar Just (dagmar.just@cecog.at). Against receipt of a signed confidentiality agreement you will be supplied with further trial-related details.

For information about all CECOG activities please visit our homepage under www.cecog.org.

Christoph Zielinski

President

Thomas Brodowicz

Director

\section{CECOG}

Dagmar Just

Central European Cooperative Oncology Group (CECOG)

Schlagergasse $6 / 6$

1090 Wien

Austria

Phone: +43/1/409 7711

Fax: +43/1/409 7726

E-mail: cecog@cecog.at

Website: www.cecog.org 